\title{
Wavelength dependent color conversion of CdSe/ZnS core/shell nanocrystals for white LEDs
}

\author{
Sedat Nizamoglu ${ }^{1,2,3}$ and Hilmi Volkan Demir ${ }^{1,2,3,4, *}$ \\ ${ }^{I}$ Nanotechnology Research Center; ${ }^{2}$ Institute of Materials Science and Nanotechnology: \\ ${ }^{3}$ Department of Electrical and Electronics Engineering; ${ }^{4}$ Department of Physics, Bilkent University, Ankara 06800 Turkey \\ *Tel:[+90](312) 2901021, e-mail: volkan@bilkent.edu.tr
}

Nanocrystals (NCs) provide narrow emission spectrum that can be conveniently tuned using quantum size effect. This ability to adjust and control emission spectrum of NCs makes them strong candidates for use in white color conversion light emitting diodes. For example, they are possibly be used for solid state lighting applications including indoor lighting, architectural lighting and scotopic street lighting, where spectrally tuned color conversion is necessary. In device research $\mathrm{CdSe} / \mathrm{ZnS}$ core/shell nanocrystals are the most commonly used ones because of their good electronic isolation coming from $\mathrm{ZnS}$ shells and the resulting high quantum efficiency (QE) (i.e., $>50 \%$ in solution) [1]. However, when these nanocrystals are integrated into the solid film, e.g., for white light generation, their in-film QE undesirably drops (despite their high QE in solution). Hence, this adversely affects the overall efficiency of the integrating devices that incorporate these NCs [2]. There have been various studies to understand the in-film optical properties of $\mathrm{CdSe} / \mathrm{ZnS}$ core/shell NCs [3-5]. However, their spectrally resolved in-film quantum efficiency (i.e., the ratio of the number of photons emitted by the nanocrystal film to the number of photons absorbed in the nanocrystal film) and their photon conversion efficiency (i.e., the ratio of the number of photons emitted by the nanocrystal film to the number of photons incident to the nanocrystal film) have not been investigated in these previous studies.

For that, in this paper we investigate spectrally resolved relative quantum efficiency and relative photon conversion efficiency of $\mathrm{CdSe} / \mathrm{ZnS}$ core/shell nanocrystals. We study the optical absorbance, photoluminescence, spectral relative quantum efficiency and spectral relative photon conversion efficiency of red-emitting $\mathrm{CdSe} / \mathrm{ZnS}$ core/shell nanocrystals with the amounts of $0.4,0.8,1.6$ and $2.4 \mathrm{nmol}$ quantum dots. We find out that both the average quantum efficiency and the average photon conversion efficiency of the nanocrystal film raise with the increasing photon excitation energy. However, in this range of nanocrystal amount, there does not exist any relation between the hybrid NC film efficiency and number of nanocrystals. Furthermore, we investigate the time dependent emission characteristics of NCs and make time-resolved spectroscopy of these NC films. As the number of in-film nanocrystals increases, the average lifetime of nanocrystals increases towards longer wavelengths. With the raising amount of NCs, the average lifetime of emission is enhanced further more and the emission of NCs is shifted further towards red. This is because of the enhanced nonradiative pumping of these nanocrystals due to the inhomogeneous size distribution. Thus, in principle for fine-tuning of the emission color of NCs for WLEDs application it is possible to tune the energy transfer (i.e., red shift) by changing the integrated number of nanocrystals.
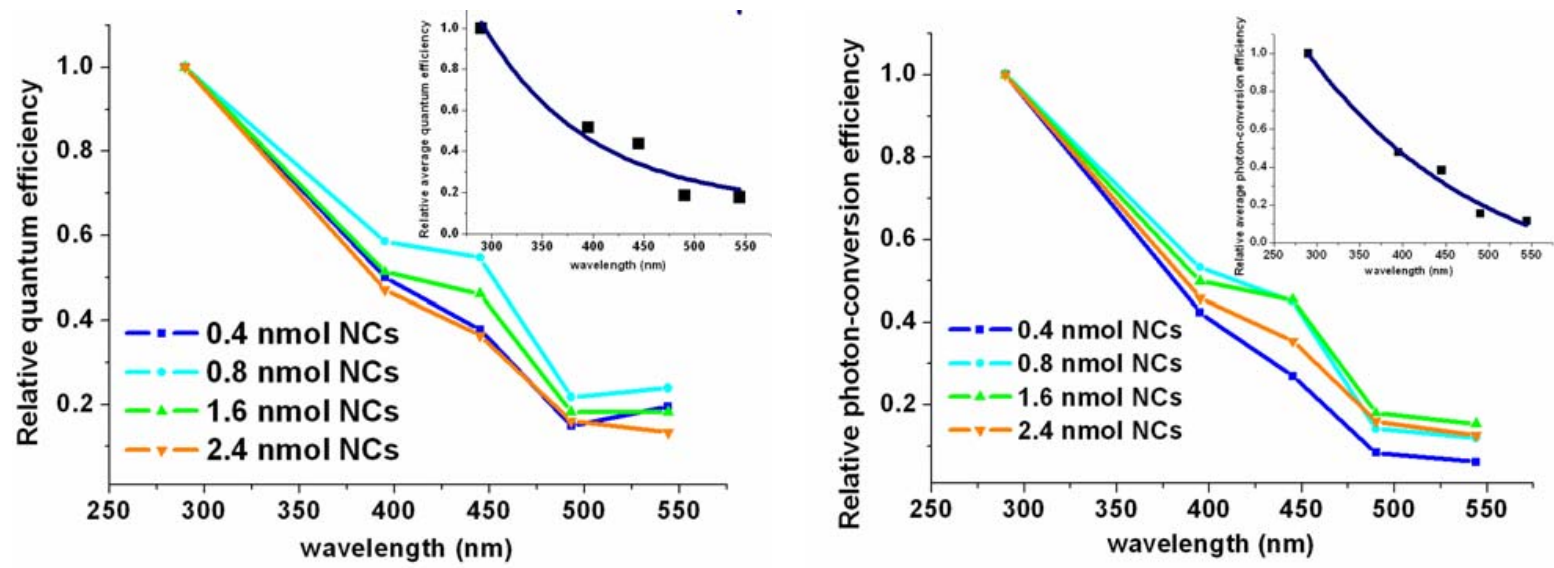

Figure 1. (a) Spectrally resolved relative quantum efficiency and (b) relative photon conversion efficiency of red emitting CdSe/ZnS core/shell nanocrystal films $\left(\lambda_{P L}\right.$ in-solution $\left.=622 \mathrm{~nm}\right)$ for the amounts of of $0.4,0.8,1.6$ and $2.4 \mathrm{nmol}$ at the excitation wavelengths of $290 \mathrm{~nm}, 395 \mathrm{~nm}, 445 \mathrm{~nm}, 493 \mathrm{~nm}$, and $544 \mathrm{~nm}$, along with the average of relative quantum efficiency and relative photon conversion efficiency in (a) and (b), respectively.

In Figure 1(a) we show the spectral relative quantum efficiency of the nanocrystal films [6]. Here the average relative QE increases towards shorter excitation wavelengths as shown in the inset of Figure 1(a). This means 
that the excitation LED platform emitting at shorter wavelengths is a better choice to pump the NCs more efficiently. The other important figure of merit for color conversion white LED application is the spectrally resolved relative photon conversion efficiency as shown in Figure 1(b). The photon conversion efficiency has also a tendency to increase with the increasing excitation energy of the incoming photon in general. On the other hand, there are discrepancies between the behavior of the photon conversion efficiency and quantum efficiency. For instance, even though the sample with $0.4 \mathrm{nmol} \mathrm{NCs} \mathrm{shows} \mathrm{the} \mathrm{highest} \mathrm{QE} \mathrm{at} 544 \mathrm{~nm}$, the photon conversion efficiency is the lowest. Here the reason is that this sample contains the fewest number of NC emitters absorbing only a portion of the incoming excitation and converting to red emission. However, the optical absorption enhances as the photon energy of the incoming photons increases and the incident photons are absorbed more and converted more efficiently to the $\mathrm{NC}$ emission. Thus, for color conversion NC-integrated LED, this experimental characterization demonstrates that it is in principle possible to achieve high color conversion with thin NC films by pumping at a shorter wavelength.
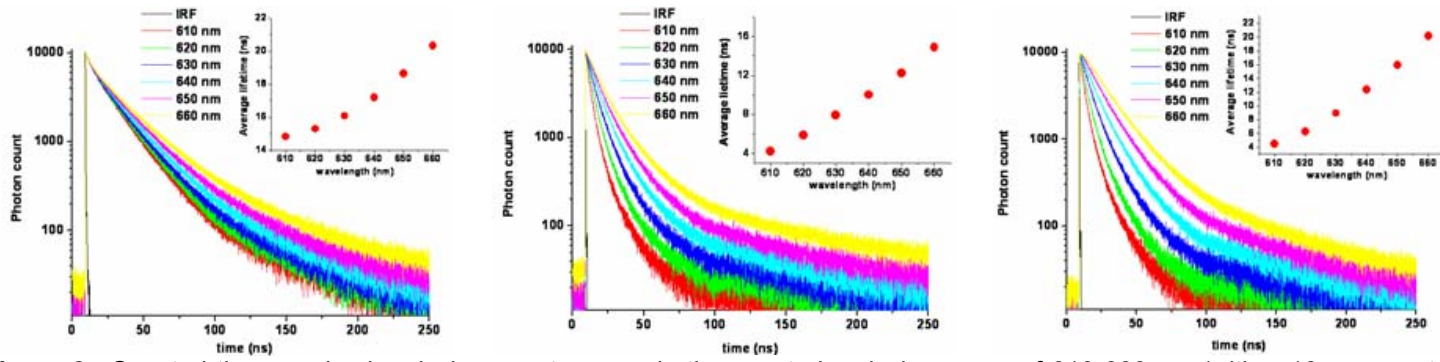

Figure 2. Spectral time-resolved emission spectroscopy in the spectral emission range of $610-660 \mathrm{~nm}$ (with a $10 \mathrm{~nm}$ spectral spacing) of (a) nanocrystals in solution, and nanocrystals in film with the amounts of (b) $0.4 \mathrm{nmol}$ and (c) $2.4 \mathrm{nmol}$.

To understand the time-dependent behavior of our NC films, we measure time-resolved photoluminescence of the NC films. For that we investigate the lifetime kinetics of nanocrystals in solution and in film with $0.4 \mathrm{nmol}$ and $2.4 \mathrm{nmol}$ of NCs between 610 and $660 \mathrm{~nm}$ with a $10 \mathrm{~nm}$ spectral spacing in Figures 2(a), 2(b) and 2(c), respectively. In Figure 2(a), the spectral dynamics of nanocrystals dispersed in solution are shown and the decay curves do not change significantly from $610 \mathrm{~nm}$ to $660 \mathrm{~nm}$. If we also look at the amplitude average lifetimes, they alter from $15 \mathrm{~ns}$ to $21 \mathrm{~ns}$, respectively, as shown in the inset of Figure 2(a). On the other hand, in the case of $0.4 \mathrm{nmol} \mathrm{NCs}$ in the solid form the time-resolved decays become more separated from each other and change drastically as going from $610 \mathrm{~nm}$ to $660 \mathrm{~nm}$ in Figure 2(b). The modification of lifetime dynamics in the case of $0.4 \mathrm{nmol} \mathrm{NCs}$ with respect to the case of NCs in solution is higher due to the environmental change from solution to air-nanocrystal where the amplitude average lifetimes change from 4 ns to $15 \mathrm{~ns}$. In addition, the interdot separation in solution is on the order of hundreds of nanometers, which is larger than the distance needed for nonradiative energy transfer among nanocrystals (i.e., around $<5 \mathrm{~nm}$ ). Thus, the nanocrystals in solution do not exhibit dipole-dipole coupling. On the other hand, in closely-packed solid films the dipole-dipole interaction becomes effective such that smaller-sized nanocrystals with wider effective bandgap give their excitonic energy to larger-sized ones with narrower bandgap due to the inhomogeneous size distribution of NCs. When the number of NCs is further increased to $2.4 \mathrm{nmol}$ as shown in Figure 2(c), the transient decay curves become even more separated from each other where the amplitude averaged lifetimes change from $4 \mathrm{~ns}$ to $20 \mathrm{~ns}$. Thus, the separation between the radiative decays at different wavelengths increases as going from solution to $0.4 \mathrm{nmol} \mathrm{NCs}$ in film and finally to $2.4 \mathrm{nmol} \mathrm{NCs}$. As a result, since the energy transfer is increased, the emission of $2.4 \mathrm{nmol} \mathrm{NCs}$ in film is shifted more to the red side of the spectrum. Therefore, varying the integrated number of nanocrystals (i.e., modifying the energy transfer) changes the emission color of NCs for color conversion LED application.

In conclusion, we presented wavelength dependent color conversion of $\mathrm{CdSe} / \mathrm{ZnS}$ core/shell nanocrystal solids. We observed that both the average quantum efficiency and the average photon conversion efficiency of the nanocrystal film increase with the increasing photon energy incident onto the nanocrystal solids. Furthermore, by changing the integrated number of NCs, we showed that it is possible to modify the red shift of emission spectrum because of the variations in the energy transfer.

Acknowledgements: This work is supported by TUBITAK 104E114, 106E020, 107E088, 107E297, and 109E002, EU MOON, EU NoE PHOREMOST. HVD acknowledges additional support from European Science Foundation European Young Investigator Award (ESFEURYI) and Turkish Academy of Sciences Distinguished Young Scientist Award (TUBA GEBIP).

Reference

[1] D. V. Talapin et al., Nano Lett. 1, 207-211 (2001).

[2] A. A. Chistyakovet et al., Laser Phys. 16, 1625 (2006).

[3] F. Gindele et al., Appl. Phys. Lett. 71, 2181 (1997).
[4] V. Biju et. al., J. Phys. Chem. C 111, 7924 (2007).

[5] V. Pilla et al., Optics Communications 280, 225 (2002).

[6] S. Nizamoglu et al., J. Appl. Phys. 105, 083112 (2009). 